\title{
Wavefront aberrations induced from biomechanical effects after customized myopic laser refractive surgery in finite element model
}

\author{
Ruirui Du \\ Nanchang Hangkong University \\ Lihua Fang ( $\nabla$ fanglh71@126.com ) \\ Nanchang Hangkong University \\ Weijian Peng \\ Nanchang Hangkong University \\ Ruizhi Yang \\ Nanchang Hangkong University \\ Shifeng Nie \\ Nanchang Hangkong University \\ Huirong Xiao \\ Nanchang Hangkong University \\ Jiahui Gong \\ Nanchang Hangkong University \\ Yu Dai \\ Nanchang Hangkong University \\ Jiahao Deng \\ Nanchang Hangkong University
}

\section{Research Article}

Keywords: Wave-front aberrations, LASIK refractive surgery, Biomechanical effects, finite element model, displacement

Posted Date: June 21st, 2021

DOI: https://doi.org/10.21203/rs.3.rs-189830/v1

License: (1) (1) This work is licensed under a Creative Commons Attribution 4.0 International License.

Read Full License 
Version of Record: A version of this preprint was published at International Ophthalmology on August 15th, 2021. See the published version at https://doi.org/10.1007/s10792-021-02003-9. 


\title{
Wavefront aberrations induced from biomechanical effects after customized myopic laser refractive surgery in finite element model
}

\author{
Ruirui Du¹, Lihua Fang1*, Weijian Peng¹, Ruizhi Yang1, Shifeng Nie¹, Huirong Xiao¹, Jiahui Gong1, \\ Yu Dai ${ }^{1}$, Jiahao Deng1 \\ ${ }^{1}$ Nanchang Hangkong University, Nanchang, 330063, China \\ ${ }^{1 *}$ Key Laboratory of Nondestructive Test (Ministry of Education), Nanchang Hangkong University , Nanchang, 86-18170938193, 330063, \\ China,.fanglh71@126.com
}

\begin{abstract}
Purpose: A customized myopic refractive surgery was simulated by establishing a finite element model of the human eye, after which we studied the wavefront aberrations induced by biomechanical effects and ablation profile after wavefrontguided LASIK surgery.

Methods: Thirty myopia patients (i.e., 60 eyes) without other eye diseases were selected. Their ages, preoperative spherical equivalent, astigmatism, and wavefront aberration were then obtained, in addition to the mean spherical equivalent error range -4 to $-8 \mathrm{D}$. Afterward, wavefront-guided customized LASIK surgery was simulated by establishing a finite element eye model, followed by the analysis of the wavefront aberrations induced by the surface displacement from corneal biomechanical effects, as well as customized ablation profile. Finally, the preoperative and induced aberrations were statistically analyzed.

Results: Comatic aberrations were the main wavefront abnormality induced by biomechanical effects, and the wavefront aberrations induced by the ablation profile mainly included coma and secondary-coma, as well as sphere and secondarysphere aberrations. Overall, the total high-order aberrations (tHOAs), total coma $\left(\mathrm{C}_{31}\right)$, and sphere $\left(C_{4}^{0}\right)$ increased after wavefront-guided customized LASIK surgery. According to our correlation analyses, coma, sphere, and total high-order aberrations were significantly correlated with decentration. Additionally, the material parameters of ocular tissue were found to affect the postoperative wavefront aberrations. When the material parameters of the sclera remained constant but those of cornea increased, the induced wavefront aberrations were reduced.
\end{abstract}

Conclusion: All biomechanical effects of cornea and ablation profile had significant effects on postoperative wavefront aberrations after customized LASIK refractive surgery; however, the effects of the ablation profile were more notorious.Additionally, the characteristics of biomechanical materials have influence on the clinical correction effect.

Keywords: Wave-front aberrations, LASIK refractive surgery, Biomechanical effects, finite element model, displacement

Acknowledgements

Thank you to everyone who has helped this study. 


\section{Introduction}

The are many forms of laser refractive surgery and LASIK ( laser-assisted in situ keratomileusis ) is one of the them. LASIK is the most commonly performed laser refractive surgery due to its short recovery times and superior safety and efficacy ${ }^{(1)}$. Vision correction through this procedure is achieved by changing the curvature of the cornea. In a conventional LASIK procedure, a hinged flap is first created using a microkeratome. The flap is folded back, and the exposed stroma is photoablated using an excimer laser. The flap is then returned to its original place to cover the treated area ${ }^{(2)}$. Jorge L. Alió et al. ${ }^{(3)}$ found that LASIK for myopia over $-10 \mathrm{D}$ is a safe procedure with myopic regression that slows down with time and a high rate of best spectacle-corrected visual acuity increase in the long-term. However, some patients that undergo LASIK surgery for moderate to severe myopia manifest optical sequelae including glares, halos, and monocular diplopia ${ }^{(4,5)}$. Moreover, many clinical studies have shown that LASIK surgery increases the likelihood of corneal higher-order aberrations ${ }^{(6,7)}$.

Some studies have reported that the correction of higher-order aberrations can improve the visual quality ${ }^{(8)}$. Particularly, wavefront-guided LASIK is a promising technique that offers the potential to correct refractive errors $^{(9)}$. C. Schallhorn et al. ${ }^{(10)}$ found that the wavefront-guided(WFG) procedures rendered similar or better refractive accuracy and uncorrected visual acuity outcomes compared to conventional LASIK. Steven Nancy J, Keir et al. ${ }^{(1)}$ found that despite an increase in higher-order aberrations, wavefront-guided LASIK yielded excellent visual acuity and contrast sensitivity. Moreover, SMILE and wavefront-guided LASIK surgery (WFG-LASIK) were found to be efficacious and safe procedures for the correction of low and moderate cases; however, WFG-LASIK allows for more predictable outcomes and better aberrometric control ${ }^{(12)}$. The finite element method has been widely used in the study of human eye biomechanics. Zhang Di et al. ${ }^{(13)}$ applied finite element analysis based on inflation test data from rabbit cornea to determine its material parameters. These parameters were then used to simulate corneal refractive surgery to study postoperative corneal deformations. Chaitanya Deenadayalu et al. ${ }^{(14)}$ used a biomechanical finite element model to study the effects of corneal modulus elasticity, flap diameter, thickness, and intraocular pressure on the refractive changes induced by the cornea flap after LASIK surgery. Eiichi Uchio et al. ${ }^{(15)}$ developed a simulation model to determine the physical and mechanical conditions that caused intraocular foreign body injuries, and the simulations were solved via finite element analysis.

Pooja et al. ${ }^{(16)}$ found that flap and cap incisions induced corneal biomechanical weakening in patients. Cornea biomechanical response to ablative surgery may significantly affect post-surgery outcomes and should be taken into account when planning customized procedures ${ }^{(17)}$. Voronin $\mathrm{G} V$ et 
al. ${ }^{(18)}$ reported that the biomechanical properties of the cornea are important for the functioning of the ocular optical system. William J Dupps et al. ${ }^{(19)}$ associated hyperopia shifts with significant thickening of the unablated peripheral stroma, and these biomechanical effects may affect the refractive outcomes of LASIK surgery. Therefore, discussing the wavefront aberrations caused by the whole- eyeball biomechanical effects after LASIK surgery is critical.

This study sought to evaluate the wavefront aberrations caused by biomechanical changes after customized LASIK surgery. A 3D finite element model of the human eye was developed to evaluate the wavefront aberrations before and after wavefront-guided LASIK surgery based on clinical measurement data. Importantly, the outcomes of this model were then compared with actual clinical results. In summary, our study proposes a novel approach to predict the effect of biomechanical changes on highorder aberrations after customized LASIK surgery, which will be of great significance for preoperative screening and postoperative visual quality optimization.

\section{Patients and Methods}

\subsection{Subjects}

In this study, the eyes of 30 potential refractive surgery candidates for correction of myopia (i.e., 60 eyes in total) were examined. None of the patients had a previous history of other ocular diseases. The age of the patients ranged from 18 to 41 years (mean 36士5.34). Fig. 1(a) shows the distribution of mean spherical equivalents ( $\mathrm{D}$ indicates the diopter). The mean spherical equivalent error range was -7.75 3.75 D, of which ranges of $-5.44 \pm 1.07 \mathrm{D}$ were observed in the right eyes and $-5.40 \pm 1.02 \mathrm{D}$ in the left eyes. Fig. 1(b) shows the astigmatic power as a scatter plot of the orthogonal components $\mathrm{J}_{0}$ and $\mathrm{J}_{45}$. The average astigmatism of the right eyes was $-0.72 \pm 0.55$ and that of the left eyes was $-0.77 \pm 0.53$. After a complete ophthalmic examination and an explanation of the nature and possible consequences of the research, the written informed consent was obtained from all patients. The wavefront aberrations of all eyes were measured using a Shack-Hartmann aberrometer under natural scotopic conditions. All measurements were repeated at least three times for each eye, and the 3 most consistent measurements were used. Wavefront aberrations were expressed as seventh-order Zernike polynomials. Patients that wore contact lenses were excluded from this study. 


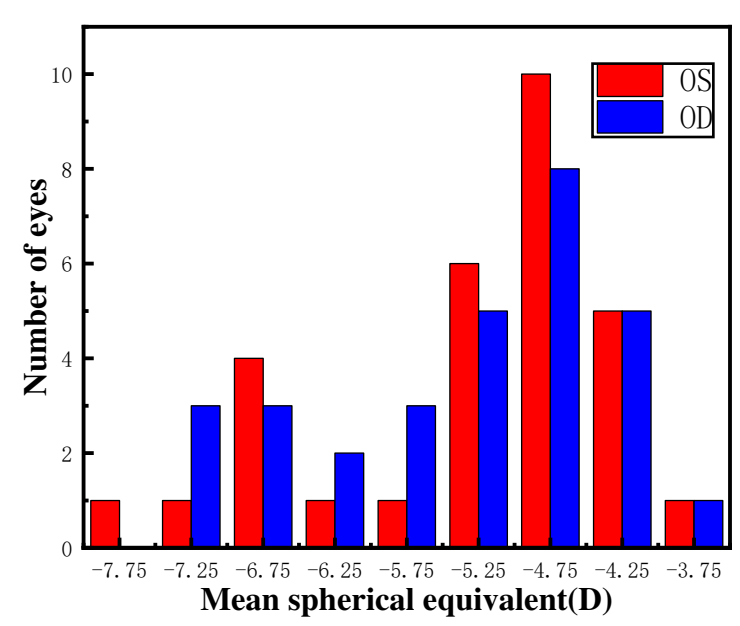

(a)

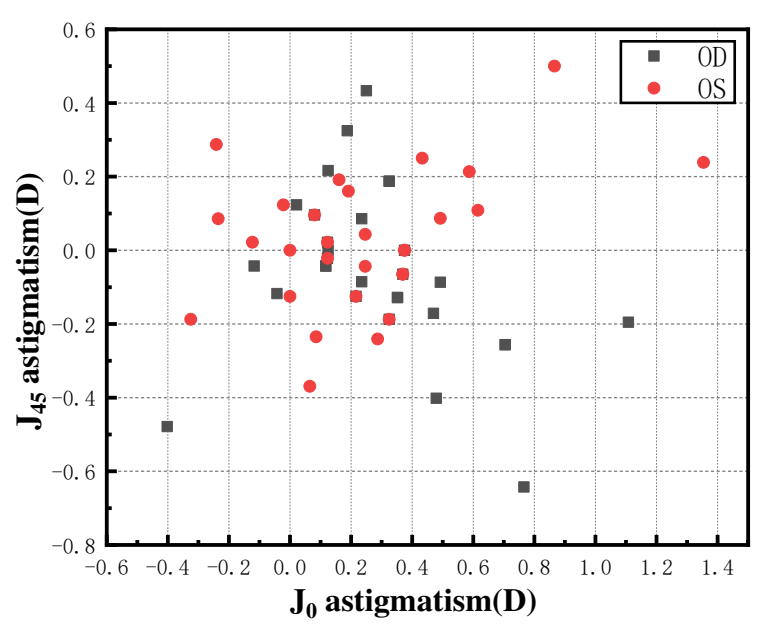

(b)

Fig. 1Frequency distributions(OD=right eyes; $\mathrm{OS}=$ left eyes).(a) represents the spherical

power of refractive error determined by the subjective refraction. (b) represents the astigmatism determined subjectively. $\mathrm{N}=60$ eyes

\subsection{Human Eye Finite Element Model}

Cornea is located at the most front of the eyeball, accounting for about 1/6 of the outer fibrous membrane of the eye. The central corneal thickness $(\mathrm{CCT})$ is $0.5 \mathrm{~mm}$, the curvature radius of anterior corneal surface is $7.7 \mathrm{~mm}$, and the curvature radius of posterior corneal surface is $6.8 \mathrm{~mm}$. Refer to the methods of building entity model in the existing literature and the cornea and sclera 3D solid morphology models were constructed by using Siemens NX.

Then there is the problem of material models. The biomechanical properties of cornea are not only related to the stable structure, but also to the materials, material model is very important. In an early study, L-Y Woo et al. ${ }^{(20)}$ reported that the cornea and sclera exhibited nonlinear material properties. The strain energy potential can be expressed as described by Equation(1):

$$
\mathrm{W}=\sum_{i=1}^{N} \frac{\mu_{i}}{\alpha_{i}}\left(\bar{\lambda}_{1}^{\alpha_{i}}+\bar{\lambda}_{2}^{\alpha_{i}}+\bar{\lambda}_{3}^{\alpha_{i}}-3\right)+\sum_{\mathrm{k}=1}^{N} \frac{1}{d_{k}}(J-1)^{2 k}
$$

where $\mathrm{W}$ represents the strain energy potential, $\bar{\lambda}_{p}$ represents the deviatoric principal stretches defined as $\bar{\lambda}_{p}=\mathrm{J}^{-\frac{1}{3}} \lambda_{p}$, and $\lambda_{p}$ indicated the principal stretches of the left Cauchy-Green tensor. $J$ represents the determinant of the elastic deformation gradient. $N, \mu_{p}, \alpha_{p}$ and $d_{p}$ are material constants.

The initial shear modulus $\mu$ is defined as:

$$
\mu=\frac{1}{2} \sum_{i=1}^{N} \alpha_{i} \mu_{i}
$$

The initial bulk modulus $k$ is defined as follows: 


$$
k=\frac{2}{d_{1}}
$$

For the purposes of this study, experimentally-derived stress versus strain data were fit to the above-described material model. We chose $n=2$ and $n=1$ as corneal and scleral fitting orders respectively. The corneal fitting parameters were: $\mu 1=3535.7 \mathrm{pa}, \alpha 1=103.51, \mu 2=3535.7 \mathrm{pa}, \alpha 2=$ 103.61; the scleral fitting parameters were: $\mu 1=30224 \mathrm{pa}, \alpha 1=182.73$. When $\mathrm{d}_{1}=0$, it was assumed that the cornea and sclera were largely indistinguishable.

By changing the thickness of residual bed, cutting depth and clinical measurement datas, the finite element model corresponding to thirty myopia patients (i.e., 60 eyes) were constructed. The thickness and diameter of the corneal flap and optical zone were $100 \mu \mathrm{m}, 8 \mathrm{~mm}$, and $6 \mathrm{~mm}$, respectively. In the calculation process, a fixed constraint was used at the bottom of sclera, the cornea and sclera were bound and connected to prevent separation and sliding, and the flap was naturally attached to the stromal layer. Cornea and sclera were divided into different-sized hexahedral meshes based on their shape and characteristics using finite element analysis and the Ansys software, Because our study focused on the cornea, the cornea meshing was relatively smaller than that of the sclera. Fig. 2 illustrates the $-4 \mathrm{D}(-4 \mathrm{DS}-$ $0.5 \mathrm{DC} \times 30)$ model in the left eyes after meshing. Moreover, the numbers of corneal nodes, corneal mesh cells, scleral nodes, and scleral mesh cells were 188767, 55854, 123698, and 33989, respectively.

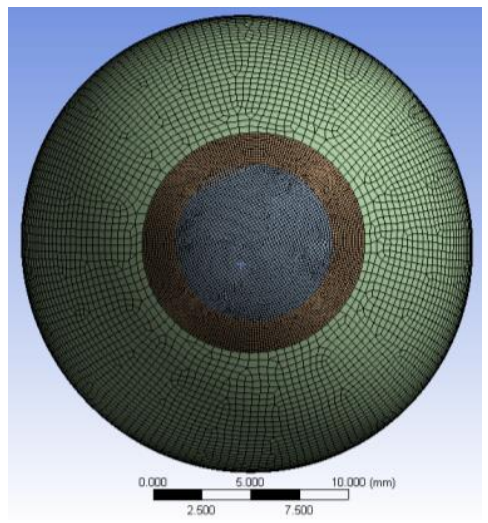

(a)

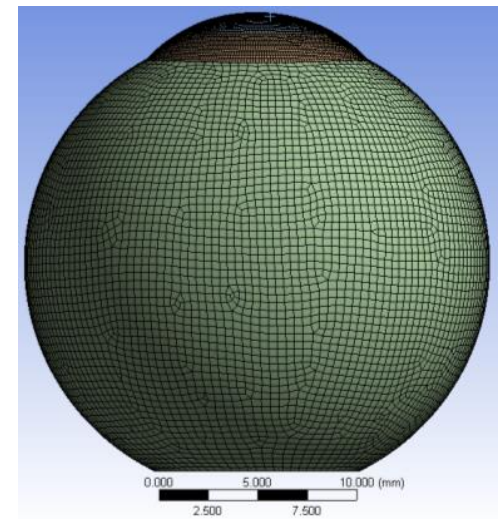

(b)

Fig. 2(a) represents the meshed whole-eye model. (b) represents the cross-section view of the $-4 \mathrm{D}(-4 \mathrm{DS}-0.5 \mathrm{DC} \times 30)$ left eye model meshes

\subsection{Customized Laser Refractive Surgery Ablation Profile}

Compared with conventional refractive surgery, wavefrond-guided ablation can reduce the preexisting higher-order aberrations and the new induced higher-order aberrations. According to the phase-conjugate principle, the ablation depth in the optical zone of any arbitrary point is given directly by the wavefront data. 


$$
D(x, y)=-\sum_{p \text { and } q} W(x, y) /(n-1)
$$

Here, the parameter $\mathrm{n}$ represents the refractive index of the cornea in visible light; $\mathrm{n}$ is 1.376 , and $(\mathrm{x}, \mathrm{y})$ depicts an arbitrary point in the optical zone on the cornea. Additionally, the wavefront $W$ is expressed as a Zernike polynomial expansion. $C_{p}^{q}$ is the Zernike coefficient.

$$
W(x . y)=\sum_{p \text { and } q} c_{p}^{q} z_{p}^{q}(x, y)
$$

Here, the parameters $\mathrm{p}$ and $\mathrm{q}$ are the radial integer index and meridional index, respectively. In this section, we aimed to structure an ablation profile for the transition zone. $\mathrm{R}$ represents the radius of the optical zone. If the width of the transition zone is $R_{\rho}$, the internal radius of the transition zone is $R$, and therefore the outer radius is $\mathrm{R}(1+\rho)$. Here, $\rho$ represents the blend coefficient of the transition zone. The ablation profile for the transition zone can be calculated as described by Equation (6):

$$
D(x, y)=D_{\mathrm{a}}(x, y) \cdot D_{b}(x, y), \quad R \leq \sqrt{x^{2}+y^{2}} \leq R(1+\rho)
$$

where $D_{\mathrm{a}}(\mathrm{x}, \mathrm{y})$ represents a blend function. Further calculations are then preformed with Equation (7). The function value is 1 at the boundary between the optical and transition zones, but the value changes to zero at the boundary between the transition zone and the untreated periphery.

$$
D_{a}(x, y)=1-\sqrt{\frac{\sin \left[\left(\frac{\pi}{R \rho}\right) \cdot\left(-\sqrt{x^{2}+y^{2}}+R+2 R \rho\right)-\frac{\pi}{2}\right]+1}{2}}
$$

In Equation (8), $D_{b}(\mathrm{x}, \mathrm{y})$ indicates the extended ablation depth in the transition zone, which is extended from the boundary value of the optical zone.

$$
D_{b}(x, y)=f\left(\frac{R x}{\sqrt{x^{2}+y^{2}}}, \frac{R y}{\sqrt{x^{2}+y^{2}}}\right)
$$

\subsection{Monte Carlo Simulating Treatment Decentration}

In this study, the Monte Carlo method was used to simulate treatment decentration. The treatment decentration number was randomly selected from a clinical study population ${ }^{(21)}$. Here, the mean transverse translation was $0.26 \pm 0.12 \mathrm{~mm}(0.04$ to $0.52 \mathrm{~mm})$. As illustrated in Fig. 3 , the mean transverse translations along the horizontal and vertical meridians in the right eyes were $-0.16 \pm 0.16$ $\mathrm{mm}(-0.46$ to $+0.13 \mathrm{~mm})$ and $-0.05 \pm 0.15 \mathrm{~mm}(-0.26$ to $0.23 \mathrm{~mm})$, respectively. On the other hand, the mean transverse translations along the horizontal and vertical meridians in the left eyes were $0.24 \pm 0.12$ $\mathrm{mm}(0.05$ to $0.49 \mathrm{~mm})$ and $-0.06 \pm 0.12 \mathrm{~mm}(-0.23$ to $0.17 \mathrm{~mm})$, respectively. 


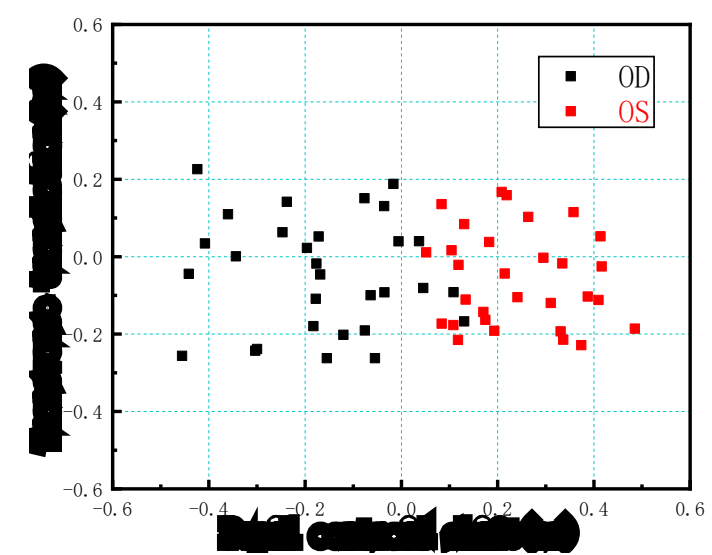

Fig. 3Transverse translation in 60 eyes $(\mathrm{OD}=$ right eyes; $\mathrm{OS}=$ left eyes $)$

\subsection{Evaluating Induced Wavefront Aberrations}

Refractive surgery can cause ocular biomechanical changes, which result in significant displacements of the anterior and posterior cornea surface due to corneal biomechanical effects. These effects can ultimately alter the refractive state of the cornea (i.e., induced wavefront aberrations after customized LASIK refractive surgery). However, the transition zone was combined with the optic zone to simulate the refractive surgery based on the ablation profile. In this study, we first discussed the wavefront aberrations caused by LASIK surgery-associated biomechanical effects on the cornea. We then studied the wavefront aberrations induced by the ablation profile of customized refractive surgery. Finally, we compared and analyzed preoperative and induced wavefront aberrations.

\section{Results}

\subsection{Wavefront Aberration Population Statistics}

Our study first analyzed the preoperative wavefront aberrations of the subjects. Fig. 4 shows the signed Zernike coefficients in the 60 eyes studied herein (i.e., 30 left eyes and 30 right eyes), including mean values and standard deviation (SD). In our study, terms like $\mathrm{C}$ with both subscript and superscript were used some places while $\mathrm{C}$ with two subscripts were used elsewhere. Because zernike polynomial functions are often referred to by their common names-magnitude/axis form. Moreover, the formula $C_{n m}=\sqrt{\left(C_{n}^{-m}\right)^{2}+\left(C_{n}^{m}\right)^{2}}$ (22) , can be used to obtain the magnitude/axis of the wavefront aberrations. 


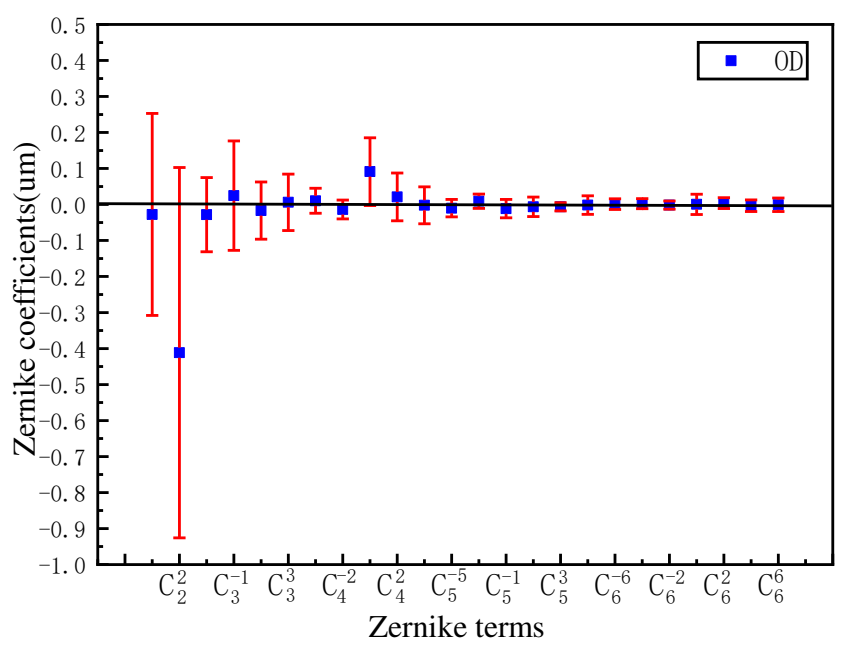

(a)

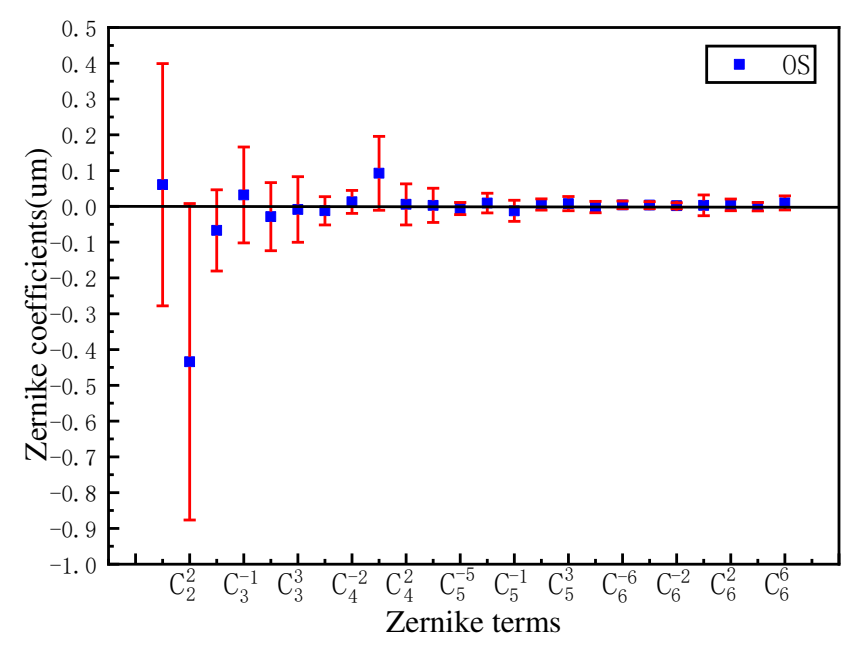

(b)

Fig.4Statistical summaries of Zernike coefficients for 30 subjects. (a) corresponds to the OD (right eyes) and (b) corresponds to the OS (left eyes). Mean values of signed wavefront aberrations coefficients are indicated by squares for all eyes, with error indicating 30 standard deviations of the population

As illustrated in Fig. 4, although slightly higher primary astigmatism $\left(C_{2}^{2}\right)$ and sphere $\left(C_{4}^{0}\right)$ values were observed $\left(C_{2}^{2}\right.$ in the right and left eyes were $-0.411 \pm 0.514 \mu \mathrm{m}$ and $-0.434 \pm 0.442 \mu \mathrm{m}$ respectively; $C_{4}^{0}$ in the right and left eyes were $0.091 \pm 0.094 \mu \mathrm{m}$ and $0.093 \pm 0.103 \mu \mathrm{m}$, respectively), both the mean and standard deviation of the other Zernike terms were close to zero. It is worth noting that, Fig. 5 also indicates that the average and SD of the Zernike terms are similar in the right and left eyes.

\subsection{Wavefront Aberrations Induced by Biomechanical Effects after Customized Refractive Surgery}

This study was based on wavefront aberration data from preoperative clinical measurements. First, the corneal ablation depths in the optical and transition zones were calculated from the customized surgical ablation profile. The corneal flap and corneal stromal surfaces were then acquired by simulating corneal stromal ablation via refractive surgery. Finally, a finite element model of the human eye was constructed. Partial corneal stroma ablation leads to decreases in corneal thickness after customized refractive surgery. The anterior and posterior surface of the cornea was displaced by loading the intraocular pressure, which resulted in corneal refractive state alterations. Under the influence of 15 $\mathrm{mmHg}$ of intraocular pressure (IOP), the displacement of the anterior and posterior surfaces of the cornea was corrected. The induced wavefront aberrations after refractive surgery were computed as the differences between the postoperative and preoperative wavefront aberrations from the displacement of the corneal surface. Moreover, the Zernike coefficients were obtained via mathematical fitting. The aberrations induced by the anterior surface were dominant, and those induced by the posterior surface were generally mild. Therefore, we concluded that the aberrations induced by the displacement of the 
anterior surface were the whole corneal surface induced wavefront aberrations after refractive surgery. Fig. 5 illustrates the aberrations induced by biomechanical effects on the corneal surface of 60 eyes.

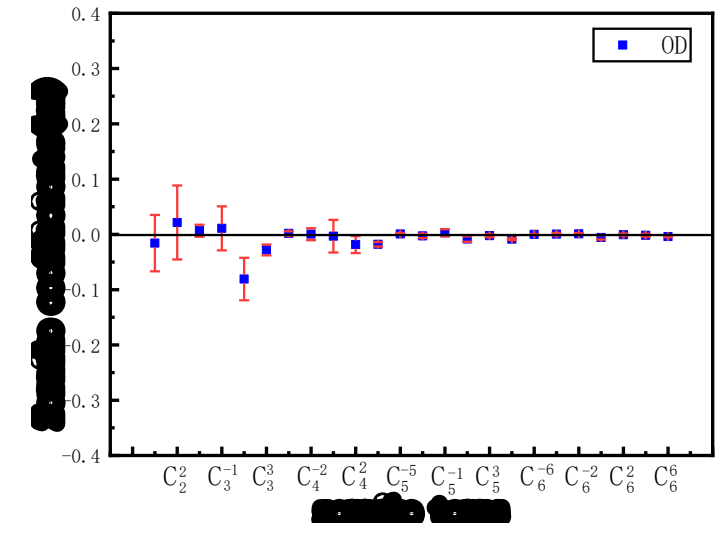

(a)

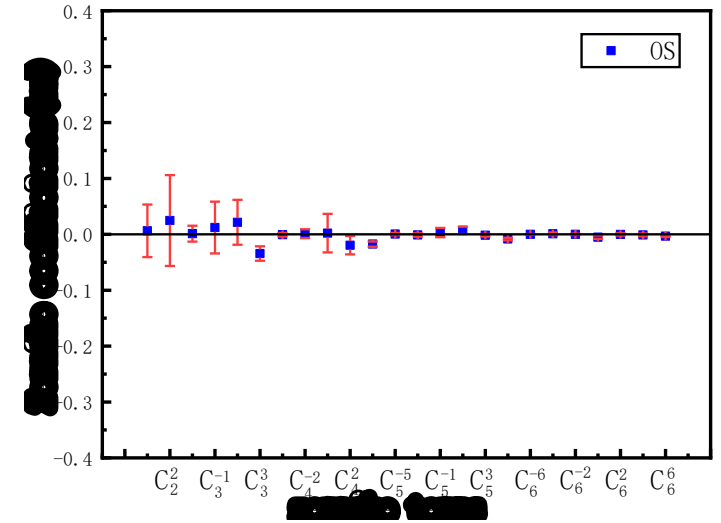

(b)

Fig.5The wavefront aberrations induced by the biomechanical effects of corneal surface were counted. (a) corresponds to the OD and (b) corresponds to the OS. Mean values of signed wavefront aberrations coefficients were indicated by squares for all eyes, with error indicating 30 standard deviations of the population

Fig. 5 shows that almost all Zernike coefficients were low in both eyes, with mean values that were close to zero. Apart from the obvious differences in comatic aberrations $\left(C_{3}^{1}\right.$ in the right and left eyes were $-0.08 \pm 0.038 \mu \mathrm{m}$ and $0.021 \pm 0.040 \mu \mathrm{m}$, respectively), the distributions of the other wavefront aberrations were largely similar.

\subsection{Effects of the Ablation Profile on Induced Wavefront Aberrations after Customized Refractive} Surgery

Using the methods described above, preoperative clinical measurement data was used to calculate the ablation depth of the corneal ablation zone, taking treatment decentration into account. The wavefront aberrations induced by the ablation profile can then be obtained by subtracting the preoperative wavefront aberrations from the optical path difference corresponding to the ablation depth. Fig. 6 displays the ablation profile-induced wavefront aberrations in 60 eyes. 


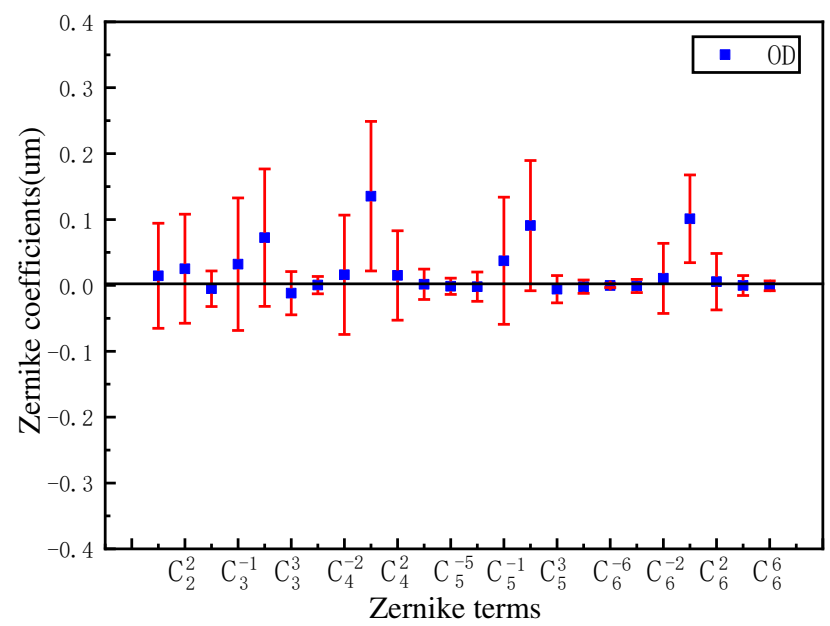

(a)

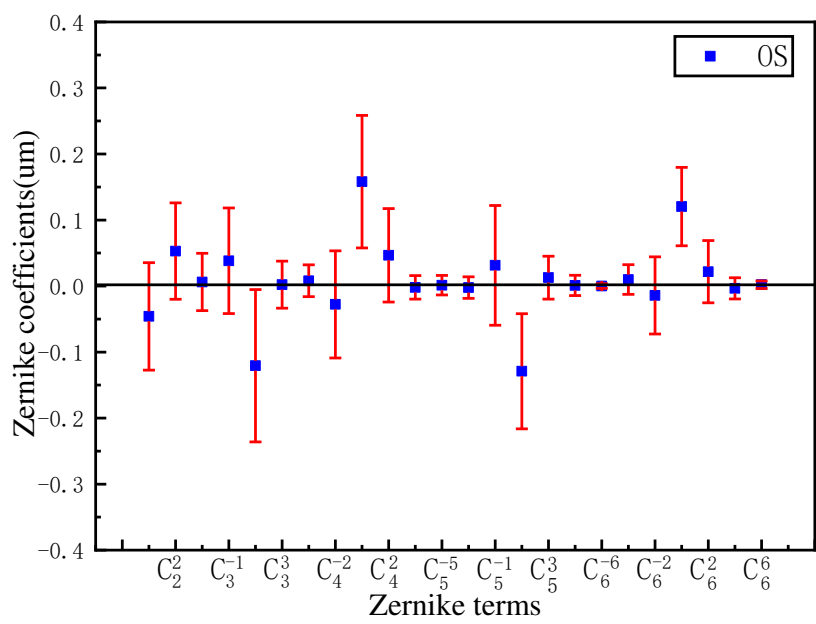

(b)

Fig.6The wavefront aberrations induced by the ablation profile of 30 subjects were calculated. (a) corresponds to the OD and

(b) corresponds to the OS. The datas were expressed in the form of mean and standard deviation

The diagram shows that, with the exception of the x-coma $\left(C_{3}^{1}\right)$ and x-secondary coma $\left(C_{5}^{1}\right)\left(C_{3}^{1}\right.$ in the right and left eyes were $0.07 \pm 0.104 \mu \mathrm{m}$ and $-0.120 \pm 0.115 \mu \mathrm{m}$, respectively; $C_{5}^{1}$ in the right and left eyes were $0.091 \pm 0.099 \mu \mathrm{m}$ and $-0.130 \pm 0.087 \mu \mathrm{m}$, respectively), there was no significant difference in the distribution of other wavefront aberrations. In addition to sphere $\left(C_{4}^{0}\right), \mathrm{x}$-coma $\left(C_{3}^{1}\right), \mathrm{x}$-secondary coma $\left(C_{5}^{1}\right)$, and $C_{6}^{0}$ values were slightly higher $\left(C_{4}^{0}\right.$ in the right and left eyes were $0.135 \pm 0.113 \mu \mathrm{m}$ and $0.158 \pm 0.100 \mu \mathrm{m}$, respectively; $C_{6}^{0}$ in the right and left eyes were $0.101 \pm 0.067 \mu \mathrm{m}$ and $0.120 \pm 0.059$ $\mu \mathrm{m}$, respectively), the rest of the wavefront aberrations were close to $0 \mu \mathrm{m}$.

\subsection{Statistical Analysis of the Preoperative and Induced Wave-front Aberrations}

Given that the other orders had little influence on the results, we mainly recorded total high-order aberrations (3 6 orders) RMS (HOARMs), y-astigmatism $\left(C_{2}^{-2}\right)$, x-astigmatism $\left(C_{2}^{2}\right)$, y-trefoil $\left(C_{3}^{-3}\right)$, x-trefoil $\left(C_{3}^{3}\right)$, y-coma $\left(C_{3}^{-1}\right)$, x-coma $\left(C_{3}^{1}\right)$, total coma $\left(C_{31}\right)$, and sphere $\left(C_{4}^{0}\right)$. The statistical values of the preoperative and induced wavefront aberrations were shown in Table 1. "I"in the table represents the preoperative wavefront aberrations, "II" refers the wavefront aberrations induced by the biomechanical effects, "III" represents the wavefront aberrations induced by the customized refractive surgery ablation profile, "IV" represents the induced aberrations (i.e., the main component of the postoperative wavefront aberrations). Statistical analyses were performed with the SPSS 25.0 software, and a P-value $<0.05$ was considered to indicate a significant difference between preoperative and induced wavefront aberrations. 
Table 1. Statistical table of preoperative and induced wavefront aberrations $(\bar{x} \pm s)$

\begin{tabular}{cccccccc}
\hline & & \multicolumn{7}{c}{ Right eyes } \\
& tHOAs & $C_{3}^{-3}$ & $C_{3}^{-1}$ & $C_{3}^{1}$ & $C_{3}^{3}$ & $\mathrm{C}_{31}$ & $C_{4}^{0}$ \\
I & $0.27 \pm 0.08$ & $-0.03 \pm 0.10$ & $0.02 \pm 0.15$ & $-0.02 \pm 0.08$ & $0.00 \pm 0.08$ & $0.15 \pm 0.07$ & $0.09 \pm 0.09$ \\
II & $0.11 \pm 0.03$ & $0.01 \pm 0.01$ & $0.01 \pm 0.04$ & $-0.08 \pm 0.04$ & $-0.03 \pm 0.01$ & $0.09 \pm 0.04$ & $-0.00 \pm 0.03$ \\
III & $0.28 \pm 0.21$ & $-0.00 \pm 0.03$ & $0.03 \pm 0.10$ & $0.07 \pm 0.10$ & $-0.01 \pm 0.03$ & $0.12 \pm 0.11$ & $0.14 \pm 0.11$ \\
IV & $0.39 \pm 0.20$ & $0.00 \pm 0.03$ & $0.04 \pm 0.10$ & $-0.01 \pm 0.11$ & $-0.04 \pm 0.03$ & $0.21 \pm 0.11$ & $0.13 \pm 0.11$ \\
P & 0.005 & 0.134 & 0.590 & 0.736 & 0.005 & 0.037 & 0.000 \\
& & & & Left eyes & & & \\
& tHOAs & $C_{3}^{-3}$ & $C_{3}^{-1}$ & $C_{3}^{1}$ & $C_{3}^{3}$ & $\mathrm{C}_{31}$ & $C_{4}^{0}$ \\
I & $0.28 \pm 0.10$ & $-0.06 \pm 0.11$ & $0.03 \pm 0.13$ & $-0.02 \pm 0.10$ & $-0.01 \pm 0.09$ & $0.15 \pm 0.08$ & $0.09 \pm 0.10$ \\
II & $0.09 \pm 0.03$ & $0.00 \pm 0.01$ & $0.01 \pm 0.05$ & $0.02 \pm 0.04$ & $-0.03 \pm 0.01$ & $0.06 \pm 0.03$ & $0.00 \pm 0.03$ \\
III & $0.33 \pm 0.19$ & $0.00 \pm 0.04$ & $0.04 \pm 0.08$ & $-0.12 \pm 0.11$ & $0.00 \pm 0.04$ & $0.15 \pm 0.12$ & $0.16 \pm 0.10$ \\
IV & $0.42 \pm 0.20$ & $0.01 \pm 0.05$ & $0.05 \pm 0.09$ & $-0.10 \pm 0.12$ & $-0.03 \pm 0.04$ & $0.20 \pm 0.12$ & $0.16 \pm 0.10$ \\
P & 0.001 & 0.002 & 0.533 & 0.015 & 0.200 & 0.012 & 0.01 \\
\hline
\end{tabular}

As indicated in Table 1, the right and left eye wavefront aberrations induced by the biomechanical effects on the corneal surface mainly manifested as increases in x-coma $\left(C_{3}^{1}\right)$. Moreover, the wavefront aberrations induced by the ablation profile mainly manifested as x-coma $\left(C_{3}^{1}\right)$ and sphere $\left(C_{4}^{0}\right)$ increase. Based on the induced aberration results, tHOAs, total coma $\left(\mathrm{C}_{31}\right)$, and sphere $\left(C_{4}^{0}\right)$ were significantly increased compared with the preoperative wavefront aberrations. In fact, the coma of II and III had a partial compensation relationship.

\section{Discussion}

\subsection{Treatment Decentration and Induced Wavefront Aberration Correlation Analysis}

During clinical surgery, the center of the pupil changed with different light conditions and sitting posture during examination and surgery, which results in laser ablation decentration. We discussed the correlation between aberrations induced by biomechanical effects and ablation profiles with decentration. Fig. 7(a) illustrates a scatter diagram of the wavefront aberrations induced by biomechanical effects on the corneal surface in 30 left eyes. Fig. 7(b) shows the scatter diagram of wavefront aberrations induced by ablation profiles. Because aberrations and decentration are two continuous variables which obey normal distribution so pearson correlation coefficients were calculated, and the correlation between the two variables was described. Table 2 summarizes the correlation coefficient analysis between wavefront aberrations and decentration, "Sig $<0.05$ " indicates that the correlation coefficients for a given subject were statistically significant (i.e., the variables were correlated). 


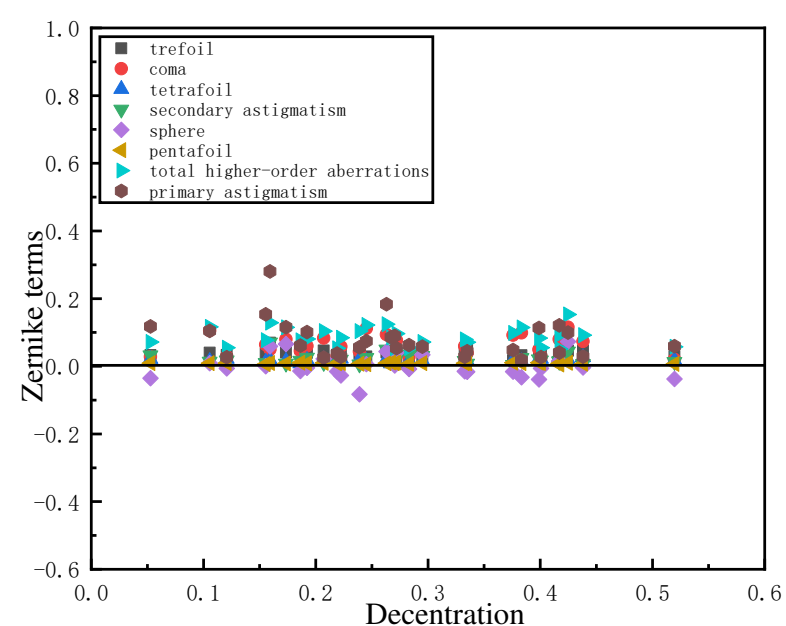

(a)

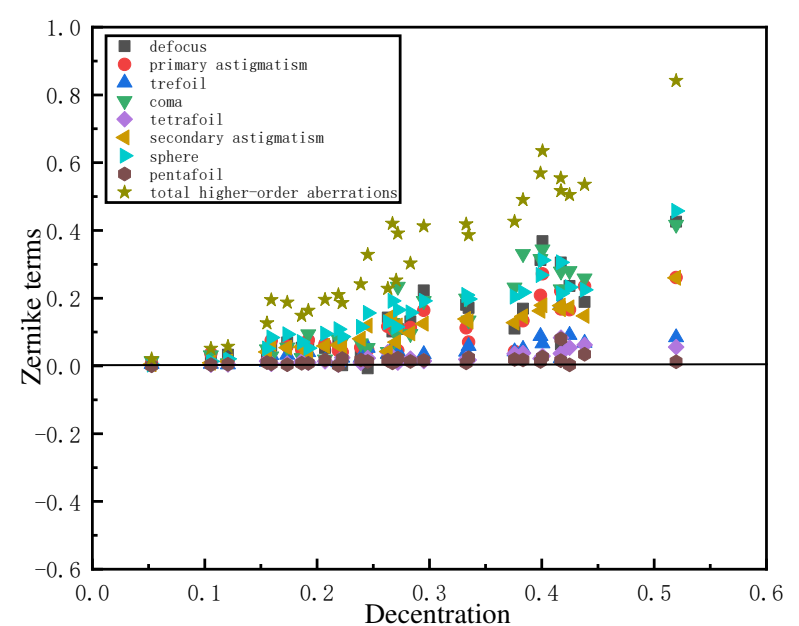

(b)

Fig.7Scatter plot of the relationship between Zernike terms and decentration. (a) represents scatter plot of the relationship between wavefront aberrations induced by biomechanical effects and treatment decentration. (b) represents scatter diagram of the relationship between wavefront aberrations induced by ablation profile and decentration

Table 2. The table of correlation between aberrations and treatment decentration

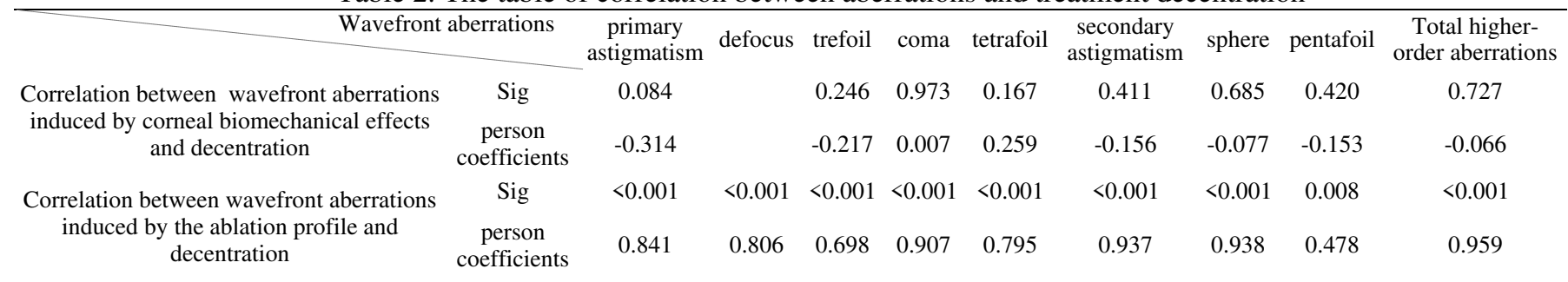

Figure. 7(a) shows that there was no correlation between wavefront aberrations and decentration, Table .2 also shows that that individual (i.e., per person) correlation coefficients between all of the wavefront aberrations and decentration were not statistically significant. The results derived from the right eyes were similar to those of the left eyes, and therefore a scatter plot and correlation coefficients were not reported. As illustrated in Fig. 7(b), there was an obvious correlation between some wavefront aberrations and decentration, and the individual coefficients are detailed in Table 2. The individual (i.e., per person) coefficients between pentafoil and decentration were the lowest. Moreover, individual coefficients for trefoil, tetrafoil and, defocus were $0.698,0.795$, and 0.806 respectively, and were found to be statistically different from the decentration values. Finally, the individual coefficients of primary astigmatism, coma, secondary astigmatism, sphere, and total higher-order aberrations ranged from 0.85 to 0.96 , and there was a highly significant difference between the aforementioned coefficients and the decentration values. The statistical results from binocular analyses were not significant difference.

\subsection{Comparison with Previous Clinical Studies}

Based on the finite element model, this study mainly analyzed the influence of biomechanical effects and ablation profile on the induction of wavefront aberrations. According to the preoperative and 
induced aberration comparison, the biomechanical effects of corneal surface alterations led to wavefront aberrations changes, and many studies have shown that refractive surgery can increase ocular wavefront aberrations. Our simulation results also indicated that tHOAs, total coma $\left(\mathrm{C}_{31}\right)$, and sphere $\left(C_{4}^{0}\right)$ were increased compared with preoperative data. Cynthia Roberts et al. ${ }^{(17)}$ suggested that the corneal biomechanical response to ablative surgery may significantly affect outcomes. William J Dup ps et al. ${ }^{(19)}$ found that the aberrations was associated with significant thickening of the unablated peripheral stroma, and these biomechanical effects may affect the refractive outcome of LASIK surgery. Fang Lihua et al.(23) also reported that among the factors affecting the incidence of higher-order aberrations after conventional laser refractive surgery, changes in corneal morphology caused by biomechanical effects had to be taken into account. Therefore, our results indicate that corneal biomechanical alteration is an important factor that influences postoperative aberrations after refractive surgery.

Additionally, the wavefront aberrations caused by the ablation profile after refractive surgery should also not be ignored. These aberrations are mainly caused by the mismatch between the treatment decentration and the optical and pupil regions. According to our preoperative and induced aberrations comparison, the wavefront aberrations induced by the ablation profile were mainly manifested as an increase in total coma. Additionally, higher-order aberrations such as x-coma $\left(C_{3}^{1}\right)$ and sphere $\left(C_{4}^{0}\right)$ also increased. According to Table 2, after customized myopic laser refractive surgery, the correlation coefficients of treatment decentration and postoperative higher-order aberrations such as primary astigmatism, coma, secondary astigmatism, sphere, and total higher-order aberrations all exhibited remarkable statistical significance. Fan Rong et al. ${ }^{(24)}$ indicated that total higher-order aberrations and coma aberrations increased significantly due to ablation-induced decentration after LASIK surgery, and they believed that decentration was among the main factors leading to vision distortion after LASIK surgery. Similar to others' report, our study indicated that treatment decentration was the main factor that led to higher-order aberrations after customized refractive surgery.

This study mainly analyzed $3^{\text {rd }}$ to $6^{\text {rd }}$ order higher-order aberrations, and higher-order aberrations can affect wavefront aberrations, of which Zernike aberration coefficients are mostly affected. MorenoBarriuso E et al. ${ }^{(25)}$ also reported that ocular aberrations ( $3^{\text {rd }}$ order and above) have a great impact on image quality. Therefore, our results were consistent with the clinical results.

\subsection{Influence of Material Parameters on the Induced Wavefront Aberrations}

The material parameters of biological tissue are key determinants of biomechanical effects. Studies have shown that the material parameters of cornea and sclera vary greatly among individuals ${ }^{(26)}$. In order to demonstrate the influence of material parameters on the wavefront aberrations of the corneal surface, 
we designed eight different cases and assigned different material parameters for cornea and sclera (Table 3 ) and the cornea and sclera were modeled with nonlinear materials. Some studies have shown that the Young's modulus of the sclera was approximately 3 to 5 times higher than that of the cornea ${ }^{(27)}$. From case 1 to 4 , the elastic range of the cornea ranged from 0.1 to $3.0 \mathrm{MPa}$, and the elastic range of the sclera ranged from 0.3 to $8.0 \mathrm{MPa}$. However, the change of corneal and scleral material parameters at the same time can only show that the material parameters have an impact on aberration, the results of cornea and sclera alone cannot be explained. So, from case 5 to 8 , the material parameters of the sclera remained constant, whereas that of the cornea ranged from 0.49 to $3.9 \mathrm{MPa}$. Therefore, the material parameters determined herein were consistent with the true values. We first selected a representative eye model from the 30 study subjects. The wavefront aberrations were then calculated using the different material parameters from the 8 cases. Finally, the displacement of the corneal surface and the Zernike coefficients were obtained. Here, $\mathrm{C}_{20}$ is defocus, $\mathrm{C}_{22}$ is primary astigmatism, $\mathrm{C}_{33}$ is trefoil, $\mathrm{C}_{31}$ is primary coma, $\mathrm{C}_{44}$ is tetrafoil, $\mathrm{C}_{42}$ is sencondary astigmatism, $\mathrm{C}_{40}$ is sphere, $\mathrm{C}_{55}$ is pentafoil, $\mathrm{C}_{53}$ is sencondary trefoil, and $\mathrm{C}_{51}$ is secondary coma. All calculations were described in section 3.4 and the results are summarized in Table 4.

Table 3. Different material parameters

\begin{tabular}{ccccccc}
\hline \multicolumn{3}{c}{ Cornea } & \multicolumn{3}{c}{ Sclera } \\
\hline Case & $\mu_{1}$ & $\alpha_{1}$ & $\mathrm{E}(\mathrm{MPa})$ & $\mu_{1}$ & $\alpha_{1}$ & $\mathrm{E}(\mathrm{MPa})$ \\
1 & 0.0084 & 13.312 & 0.108 & 0.0254 & 23.716 & 0.325 \\
2 & 0.0109 & 32.192 & 0.325 & 0.0289 & 63.49 & 0.974 \\
3 & 0.0120 & 88.684 & 0.974 & 0.0302 & 182.73 & 2.923 \\
4 & 0.0124 & 258.09 & 2.923 & 0.0307 & 540.4 & 8.770 \\
\hline 5 & 0.0114 & 46.321 & 0.487 & & & \\
6 & 0.0120 & 88.684 & 0.974 & 0.0302 & 182.73 & 2.923 \\
7 & 0.0123 & 173.39 & 1.949 & & & \\
8 & 0.0125 & 342.8 & 3.898 & & & \\
\hline
\end{tabular}

Table 4. Summary of Induced Zernike wavefront aberrations on the Cornea

\begin{tabular}{cccccccccc}
\hline \multicolumn{10}{c}{ Wave-Front Aberrations } \\
Case & \multicolumn{8}{c}{ Low-Order Aberrations $(\mu \mathrm{m})$} & \multicolumn{7}{c}{ High-Order Aberrations $(\mu \mathrm{m})$} \\
\cline { 2 - 11 } & $\mathrm{C}_{22}$ & $\mathrm{C}_{20}$ & $\mathrm{C}_{31}$ & $\mathrm{C}_{44}$ & $\mathrm{C}_{42}$ & $\mathrm{C}_{40}$ & $\mathrm{C}_{55}$ & $\mathrm{C}_{53}$ & $\mathrm{C}_{51}$ \\
1 & 0.644 & -3.666 & 0.185 & 0.065 & 0.024 & 0.179 & 0.020 & 0.005 & 0.030 \\
2 & 0.302 & -1.541 & 0.151 & 0.046 & 0.023 & 0.141 & 0.016 & 0.006 & 0.020 \\
3 & 0.098 & -0.857 & 0.079 & 0.021 & 0.015 & 0.063 & 0.007 & 0.004 & 0.007 \\
4 & 0.077 & -0.626 & 0.031 & 0.008 & 0.008 & 0.034 & 0.003 & 0.001 & 0.002 \\
\hline 5 & 0.274 & -1.719 & 0.122 & 0.036 & 0.018 & 0.071 & 0.012 & 0.006 & 0.015 \\
6 & 0.098 & -0.857 & 0.079 & 0.021 & 0.015 & 0.063 & 0.007 & 0.004 & 0.007 \\
7 & 0.034 & -0.636 & 0.046 & 0.011 & 0.011 & 0.061 & 0.004 & 0.002 & 0.002 \\
8 & 0.014 & -0.624 & 0.025 & 0.005 & 0.009 & 0.056 & 0.002 & 0.001 & 0.000 \\
\hline
\end{tabular}

Table 4 summarizes the induced wavefront aberrations derived from corneal surface displacement were shown in the eight studied cases. Material parameters had a significant influence on hyperopic shift; however, the effects on higher-order aberrations were less than $0.19 \mu \mathrm{m}$. From case 1 to case 4 , we 
concluded that the elastic modulus of the cornea and sclera increased simultaneously, the low-order aberrations decreased after refractive surgery, and the hyperopic shift $\left(\mathrm{C}_{20}\right)$ decreased rapidly. From case 5 to case 8, when scleral elasticity remained constant and corneal elasticity increased, the hyperopic shift, astigmatism, and coma decreased, and the variation of spherical aberrations was also decreased. Roy A $S$ et al. ${ }^{(28)}$ found that differences in the corneoscleral stiffness relationship affect simulated outcomes and may be a source of indiviual variation in refractive surgery outcomes. Therefore, we believe that changes in ocular tissue material parameters will likely affect the occurrence and characteristics of postoperative residual wavefront aberrations.

\section{Other Thoughts}

In this study, the finite element model was based on classical Gullstrand eye model and the Munnerlynn-based profile, in which the individual clinical data of 30 potential refractive surgery candidates were not considered. But, our results represent the average of 30 patients. Additionally, the effects of hinge chord length were considered and we set it to a constant value of $4 \mathrm{~mm}$, but this size of hinge chord length may not be optimal. In fact, some studies have shown that the hinge chord length had an effect on the higher-order aberrations after LASIK surgery. Additionlly, the model without considering the epithelial layer and transitional zone. The epithelial layer plays an important role in the biomechanical properties of cornea, Sano et al. ${ }^{(29)}$ indicated that the protrusion of abnormal substance in corneal elastic layer after PRK, this may be due to the destruction of corneal epithelial integrity.

In addition, the material parameters of cornea and sclera were from previous publications. Our study found that the material properties of eye tissues had an important effect on the biomechanical properties. Chaitanya Deenadayalu et al. ${ }^{(30)}$ also found that the elastic modulus had a great influence on the hyperopic shift. In our study, although the material parameters of the sclera were set to be 3 times that of the cornea, the actual relationship of scleral and corneal material parameters must be different from this situation. Furthermore, previous studies have shown that there were remarkable individual differences in the material parameters of eye tissue. Therefore, it is very important to measure the material parameters of eye tissue in vivo for the finite element analysis.

The corneal surface displacement included not only the displacement through the Z-axis, but also

the displacement through both the $\mathrm{X}$ - and Y-axis. In this research, these displacements were all considered and the calculation of wavefront aberrations were guaranteed to be accurate. Additionally, the individual differences in intraocular pressure were not considered. During the simulation, the intraocular pressure was set to be a constant value of $15 \mathrm{mmHg}$, the prestressing stress of zero 
intraocular pressure is not discussed. We also did not discuss the aberrations of low and high intraocular pressure loading respectively.

After customized LASIK refractive surgery, the wave-front aberrations of clinical measurements may be derived from the postoperative recovery, wound healing, and other external factors. Ikuko Toda et al. ${ }^{(31)}$ indicated that patients undergoing LASIK develop dry eye with compromised tear function for at least 1 month after surgery, this may also be related to postoperative wave-front aberrations. Additionally, there are many factors affect the postoperative biomechanical properties including the microstructure of corneal tissue and different surgical procedures.

In fact, the simulation results of refractive surgery may be deviate slightly from the actual clinical results, our goal is to simulate the clinical situation as much as possible and reduce this bias. In followup work, a personalized finite element eye model was constructed based on the clinic data and material parameters measured in vivo. Then, the biomechanical effects of hinge chord length, material parameters and, intraocular pressure on induced wavefront aberrations should be quantitatively analyzed. Therefore, the finite element analysis results will be more accurate and the biomechanical characteristics of human eye was better understood.

\section{Conclusion}

In this study, a human eye finite element model was used to simulate customized LASIK refractive surgery. Moreover, we studied the wavefront aberrations induced by the biomechanical effects on the anterior and posterior corneal surfaces and the ablation profile. Our results demonstrated that corneal biomechanical alteration resulted mainly in comatic aberrations. Moreover, the wavefront aberrations induced by ablation profiles mainly included coma, secondary-coma, sphere, and secondary-sphere aberrations. Overall, the total high-order aberrations (tHOAs), total coma $\left(\mathrm{C}_{31}\right)$, and sphere $\left(C_{4}^{0}\right)$ were increased after customized LASIK surgery. Additionally, our statistical analyses demonstrated that coma, sphere, and tHOAs were significantly correlated with decentration. Furthermore, the materials parameters of ocular tissue can affect the postoperative wavefront aberrations, and the harder the cornea, the smaller the aberrations. Specifically, the induced wavefront aberrations were ameliorated when the material parameters of the sclera remained constant while those of the cornea increased. Both corneal biomechanical effects and ablation profile have significant effects on postoperative wavefront aberrations after customized LASIK refractive surgery; however, the effects associated with ablation profile are more notorious.

\section{Compliance with Ethical Standards}


Disclosure of potential conflicts of interest:The authors have no relevant financial or non-financial interests to disclose.

Research involving Human Participants and/or AnimalsDisclosures and declarations:Our research involving human paratipants and no animals.

Informed consent:Informed consent was obtained from all individual participants included in the study. Declarations

Conflicts of interest/competing interests:No relevant financial or non-financial interests.

Funding:Supported by the Natural National Science Foundation of China (NSFC) (61465010 \& 81873684), the National Key Research and Development Program of China (2018YFE0115700) and Jiangxi Nature Science Foundation (20192BAB207035).

Ethics Approval:Approval was obtained from the ethics committee of Tianjin Eye Hospital.

Data Availability:Data are transparent.

Code and/or material availability:In the process of research we use MATLAB, ANSYS and NX, and have program.

Animal Research:This study does not involve the animal.

Consent to Participate:Informed consent was obtained from all individual participants included in the study.

Consent to Publish:The participant has consented to the submission of the case report to the journal.

\section{Author' contribution statements}

Du Ruirui: Conceptualization, Methodology, Software, Formal analysis, Writing - Original Draft, Data Curation, Supervision. Fang Lihua: Conceptualization, Visualization, Resources, Writing - Review \& Editing, Funding acquisition, Project administration, Project administration. Peng Weijian: Conceptualization, Data Curation, Validation. Yang Ruizhi: Software, Visualization. Nie Shifeng: 
Software, Methodology. Xiao Huirong: Project administration. Gong Jiahui: Software, Formal analysis.

Dai Yu: Software, Data Curation. Deng Jiahao: Software, Data Curation.

\section{References}

1. C. Song-En, Chan, C.-y., Tommy. Application of small-incision lenticule extraction in myopic astigmatic correction[J],(2019).

2. M.H. Vesaluoma, Perez-Santonja, J.J., Petroll, W.M., Linna, T., Tervo, T.J.I.O., Science, V. Corneal stromal changes induced by myopic LASIK[J], Investigative Ophthalmology \& Visual ence, 41(2): 369-376,(2000).

3. J.L. Alió, Muftuoglu, O., Ortiz, D., Pérez-Santonja, J.J., Artola, A., Ayala, M.J. et al. Ten-year Follow-up of Laser In Situ Keratomileusis for High Myopia[J], Am J Ophthalmology, 145(1): 5564.e51,(2008).https://doi.org/10.1016/j.ajo.2007.08.035

4. N.I. Fan-Paul, Li, J., Miller, J.S., Florakis, G.J.J.S.o.O. Night Vision Disturbances After Corneal Refractive Surgery[J], Survey of Ophthalmology, 47(6): 533-546,(2002).https://doi.org/10.1016/S0039-6257(02)00350-8 
5. P.S. Hersh, Steinert, R.F., Ophthalmology, S.F.B.J. Photorefractive keratectomy versus laser in situ keratomileusis: Comparison of optical side effects[J], Ophthalmology, ,(2000).https://doi.org/10.1016/S0161-6420(00)00059-2

6. S. Ninomiya, Maeda, N., Kuroda, T., Fujikado, T., Tano, Y.J.S.i.O. Comparison of ocular higher-order aberrations and visual performance between photorefractive keratectomy and laser in situ keratomileusis for myopia[J], Seminars in Ophthalmology, 18(1): 29-34,(2003).https://doi.org/10.1076/soph.18.1.29.14069

7. K.J.J.o.R.S. Pesudovs. Wavefront aberration outcomes of LASIK for high myopia and high hyperopia[J], Journal of Refractive Surgery, 21(5): S508,(2005).https://doi.org/10.3928/1081-597X-20050901-18

8. D.R. Williams, Yoon, G.Y., Porter, J., Guirao, A., Cox, I.J.J.o.R.S. Visual benefit of correcting higher order aberrations of the eye[J], Journal of Refractive Surgery, 16(5): S554-559,(2000).

9. M. Mrochen, Kaemmerer, M., Seiler, T.J.J.o.O., Ophthalmology. Clinical results of wavefront-guided laser in situ keratomileusis 3 months after surgery[J], Journal of Optometry \& Ophthalmology, 27(2): 201207,(2001).https://doi.org/10.1016/S0886-3350(00)00827-0

10. S.C. Schallhorn, Farjo, A.A., Huang, D., Wachler, B.S.B., Sugar, A.J.O. Wavefront-guided LASIK for the correction of primary myopia and astigmatism a report by the American Academy of Ophthalmology[J], Ophthalmology, 115(7): 1249-1261,(2008).https://doi.org/10.1016/j.ophtha.2008.04.010

11. N.J. Keir, Trefford, S., Jones, L.W., Desmond, F.J.J.o.R.S. Wavefront-guided LASIK for myopia: effect on visual acuity, contrast sensitivity, and higher order aberrations[J], Journal of Refractive Surgery, 25(6): 524533,(2009).https://doi.org/10.3928/1081597X-20090512-06

12. M.A. Khalifa, Ghoneim, A., Shaheen, M.S., Aly, M.G., Piñero, D.P.J.J.o.R.S. Comparative Analysis of the Clinical Outcomes of SMILE and Wavefront-Guided LASIK in Low and Moderate Myopia[J], Journal of Refractive Surgery, 33(5): 298-304,(2017).https://doi.org/10.3928/1081597X-20170222-01

13. S.T. Zhang D I , Zhang H, et al. THE SIMULATION STUDY ON THE DEFORMATION OF RABBIT CORNEA AFTER REFRACTIVE SURGERY WITH DIFFERENT CUTTING THICKNESS[J], Journal of Mechanics in Medicine and Biology, 17(2): 1750118,(2017).https://doi.org/10.1142/S0219519417501184

14. C. Deenadayalu, Mobasher, B., Subby, P., Rajan, D., Gary, P., Hall, W.J.J.o.R.S. Refractive change induced by the LASIK flap in a biomechanical finite element model[J], Journal of Refractive Surgery, 22(3): 286292,(2006).https://doi.org/10.3928/1081-597X-20060301-15

15. E. Uchio, Ohno, S., Kudoh, J., Aoki, K., Kisielewicz, L.T.J.B.J.o.O. Simulation model of an eyeball based on finite element analysis on a supercomputer[J], British Journal of Ophthalmology, 83(10): 11061111,(1999).https://doi.org/10.1136/bjo.83.10.1106

16. Pooja, Khamar, Rohit, Shetty, Ravish, Vaishnav et al. Biomechanics of LASIK Flap and SMILE Cap: A Prospective, Clinical Study[J], Journal of Refractive Surgery, ,(2019).https://doi.org/10.3928/1081597X-20190319$\underline{01}$

17. C.J.J.o.R.S. Roberts. Biomechanics of the cornea and wavefront-guided laser refractive surgery[J], Journal of Refractive Surgery, 18(5): S589,(2002).

18. G.V. Voronin, Oftalmologii, I.A.B.J.V. Changes in biomechanical properties of the cornea after keratorefractive surgery[J], Vestnik oftalmologii, 135(4): 108,(2019).https://doi.org/10.17116/oftalma2019135041108

19. W.J. Dupps, Surgery, C.R.J.J.o.R. Effect of acute biomechanical changes on corneal curvature after photokeratectomy[J], Journal of Refractive Surgery, 17(6): 658-669,(2001).

20. L.Y. Woo, Kobayashi, A.S., Schlegel, W.A., Lawrence, C.J.E.E.R. Nonlinear material properties of intact cornea and sclera[J], Experimental Eye Research, 14(1): 29-39,(1972).https://doi.org/10.1016/0014-4835(72)90139-X

21. L. Wang, Koch, D.D.J.J.C.R.S. Residual higher-order aberrations caused by clinically measured cyclotorsional misalignment or decentration during wavefront-guided excimer laser corneal ablation[J], J Cataract Refract Surg, 34(12): 2057-2062,(2008).https://doi.org/10.1016/j.jcrs.2008.08.015

22. I. Standards. Ophthalmic Optics And Instruments - Reporting Aberrations Of The Human Eye[J],

23. M.W. Fang L, Wang Y, Dai Y, Fang Z. . Theoretical analysis of wave-front aberrations induced from conventional laser refractive surgery in a biomechanical finite element model, Invest Ophthalmol Vis Sci, 0 (0) : 28669,(2020).

24. R. Fan, Yulan, D.I., Qiu, Y., Zhai, G., Zhe, Q.U., Yaoyu, L.I.J.J.o.I. et al. Ablation induced decentration in postLASIK eyes[J], Journal of Intelligent \& Fuzzy Systems, 28(4): 1885-1897,(2011).

25. E. Moreno-Barriuso, , J., Marcos, S., Navarro, R., Llorente, L., Barbero, S.J.I.O.V.S. Ocular aberrations before and after myopic corneal refractive surgery: LASIK-induced changes measured with laser ray tracing[J], Invest Ophthalmol Vis, 42(6): 1396-1403,(2001).

26. W.-P. Shih, Wang, et al. . A corneal elastic dynamic model derived from Scheimpflug imaging technology[J], Ophthalmic \& Physiological Optics the Journal of the British College of Ophthalmic Opticians, ,(2015).

27. P.J. Shih, Guo, Y.R.J.J.o.t.A.S.o.A. Resonance frequency of fluid-filled and prestressed spherical shell—A model of the human eyeball[J], Journal of the Acoustical Society of America, 139(4): 1784,(2016).https://doi.org/10.1121/1.4945733 
28. A.S. Roy, Dupps, W.J.J.J.o.R.S. Effects of Altered Corneal Stiffness on Native and Postoperative LASIK Corneal Biomechanical Behavior: A Whole-Eye Finite Element Analysis[J], Journal of Refractive Surgery, 25(10): 875887,(2009).https://doi.org/10.3928/1081597X-20090917-09

29. Sano, Yuta. Changes in descemet membrane and endothelium after corneal epithelial abrasion alone and with photorefractive keratectomy in rabbits[J], Archives of Ophthalmology, 114(9): 1105,(1996).https://doi.org/10.1001/archopht.1996.01100140307009

30. C. Deenadayalu, Mobasher, B., Subby, P., Rajan, D., Gary, P., Hall, W.J.J.o.R.S. Refractive change induced by the LASIK flap in a biomechanical finite element model[J], Journal of Refractive Surgery, 22(3): 286292,(2006).https://doi.org/10.3928/1081-597X-20060301-15

31. I. Toda, Asano-Kato, N., Komai-Hori, Y., Tsubota, K.J.A.J.o.O. Dry eye after laser in situ keratomileusis[J], Am J Ophthalmology, 132(1): 1-7,(2001).https://doi.org/10.1016/S0002-9394(01)00959-X 